The Astrophysical Journal, 643:266-275, 2006 May 20

(C) 2006. The American Astronomical Society. All rights reserved. Printed in U.S.A.

\title{
SHORT GAMMA-RAY BURSTS WITH EXTENDED EMISSION
}

\author{
J. P. Norris AND J. T. Bonnell \\ Astroparticle Physics Laboratory, NASA/Goddard Space Flight Center, Greenbelt, MD 20771 \\ Received 2005 October 24; accepted 2006 January 15
}

\begin{abstract}
The recent association of several short gamma-ray bursts (GRBs) with early-type galaxies with low star formation rates demonstrates that short bursts arise from a different progenitor mechanism than long bursts. However, since the duration distributions of the two classes overlap, membership is not always easily established. The picture is complicated by occasional softer, extended emission lasting tens of seconds after the initial spikelike emission comprising an otherwise short burst. Using the large BATSE sample with time-tagged event (TTE) data, we show that the fundamental defining characteristic of the short-burst class is that the initial spike exhibits negligible spectral evolution at energies above $\sim 25 \mathrm{keV}$. The behavior is nearly ubiquitous for the 260 bursts with $T_{90}<2 \mathrm{~s}$ for which the BATSE TTE data type completely included the initial spike. We find this same signature-negligible spectral lag - for six Swift BAT short bursts and one HETE-2 short burst. We also analyze a small sample of "short" BATSE bursts - those with the most fluent, intense extended emission. The same lack of evolution on the pulse timescale obtains for the extended emission in the brighter bursts for which significant measurements can be made. We also show that the dynamic range in the ratio of peak intensities, spike:extended, is $\sim 10^{4}$. However, for our BATSE sample the total counts fluence of the extended component equals or exceeds that in the spike by a factor of several. A high Lorentz factor, $\sim 500-1000$, might explain the negligible lags.
\end{abstract}

Subject heading: gamma rays: bursts

\section{INTRODUCTION}

In the Konus catalog of short gamma-ray bursts, Mazets et al. (2002) discuss "short" bursts, which are accompanied by lowintensity, extended emission persisting for tens of seconds after the initial spikelike, harder emission. In the Konus sample the extended component is detected individually in 11 of 130 short bursts. Frederiks et al. (2004) summarize this work and report its presence when tens of bursts are averaged. A similar spectrally soft, extended component was detected in the aggregate for Burst and Transient Source Experiment (BATSE) bursts and interpreted as the onset of a weak hard X-ray afterglow (Lazzati et al. 2001; Connaughton 2002).

Several questions remain about the two components and their causal connection. One of the most fundamental, as mentioned by Mazets et al., is how to distinguish short bursts from the more prevalent long bursts, given that some short bursts have extended emission, whereas some long bursts have only one or two pulse episodes - where is the dividing line? Also, it is unclear if all short bursts have extended emission at some level — what is the dynamic range in intensity of the extended emission relative to the spike emission? Besides being softer, the temporal and spectral character of the extended emission at the pulse level remained to be elucidated - what is the character of the extended emission's pulses, if it can be characterized as such?

The delineation between the two recognized classes of bursts has often been made in a two-dimensional diagram of spectral hardness versus duration, in which short bursts appear slightly harder than long bursts (Kouveliotou et al. 1993; Meegan et al. 1996). However, the observed average difference in hardness may be at least partially attributable to obvious selection effects: In any GRB trigger algorithm the instrument's effective area as a function of energy limits which bursts may trigger. For instance, Sakamoto (2006) showed that the sample of Konus short bursts has a lower hardness ratio than the BATSE sample. Also, trigger algorithms often use multiple energy bands and accumulation timescales; a sample of long bursts will tend to profit more from the latter measure than can the short-burst sample. Recall that the first $2 \mathrm{~s}$ of long bursts are on average as spectrally hard as short bursts, after which long bursts tend to soften (Ghirlanda et al. 2004). Moreover, brighter long bursts are harder (Mallozzi et al. 1995). In the BATSE sample we would not have detected some of the long bursts near threshold if they did not commence as spectrally hard. Thus, the distribution of hardnesses in both parent populations is probably much different than in detected samples (cf. $\mathrm{X}$-ray flashes [XRFs] and GRBs).

In this work, we are primarily concerned with the dichotomy in the duration dimension. In fact, this primary defining characteristic of short bursts is insufficient to cleanly isolate a physical, rather than a one-dimensional, phenomenological class. Long and short bursts' quasi-lognormal distributions overlap, defining a valley near $2 \mathrm{~s}$, with some members of each group intruding slightly into the other's duration domain. The degree of intrinsic overlap, and the extent to which extrinsic factors smear the two distributions is not yet established, but the important smearing factors are (at least) four: The difference in signal-to-noise ratio $(\mathrm{S} / \mathrm{N})$ levels between the most intense and dimmest BATSE bursts (otherwise identical) gives rise to a factor of $\sim 2$ range in measured duration (Bonnell et al. 1997). Distant long bursts $(z \sim 2-10)$ detectable by Swift (Lamb \& Reichart 2000) are time dilated relative to bursts at $z \approx 1$ by factors of $2-5$. For short bursts the relative dilation factors will usually be less than 2, assuming those detectable by Swift and the High Energy Transient Explorer (HETE) continue to lie predominantly at redshifts $z<1$. Therefore, this effect should increase the separation between the classes. Redshift of the spectral energy distribution operates in the reverse direction of time dilation; the dependence of individual pulse durations and spectral lags on energy is roughly $\sim E^{-0.35}$ (Norris et al. 1996; Fenimore et al. 1995). Thus, the energy band of observation is a factor in measured durations of short bursts, which tend to consist of one to just a few pulses (Norris et al. 2001, hereafter NSB01). Moreover, we do not necessarily detect the 
shortest bursts, since each instrument algorithm requires a minimum fluence to trigger, introducing truncation effects on the short end of the duration distribution (Lee \& Petrosian 1996).

These questions are brought into focus and made more pressing with the recent detections of short bursts by Swift and by HETE-2, two with extended emission. Before the Swift era the distance scale(s) and progenitor type(s) of short gamma-ray bursts (GRBs), those persisting for less than $\sim 2 \mathrm{~s}$, were still uncertain. Only the isotropic distribution of short GRBs - like that of long GRBs - as revealed by the large BATSE sample, provided a substantive argument in favor of their cosmological distances (Briggs et al. 1996). To date (2005 December 10) 10 short bursts have been detected and localized by Swift and HETE-2, and four of these bursts have redshifts determined from their host galaxy or apparent membership in a galaxy cluster. Thus, the major question concerning the distance scale for short bursts is qualitatively answered, the redshifts for these bursts being $z=0.16,0.226,0.258$, and 1.8 (Fox et al. 2005; Bloom et al. 2006; Gehrels et al. 2005; Barthelmy et al. 2005b; M. D. Gladders \& E. Berger 2006, in preparation). In two cases (GRBs $050509 \mathrm{~b}$ and 050724 ) the burst has been clearly associated with an elliptical galaxy with a low star formation rate. The case for coalescing neutron star (NS)-NS or NS-black hole $(\mathrm{BH})$ binaries as the progenitor populationrather than the massive stellar collapse model for long bursts - is strengthened by an analysis of relatively small error boxes of several short bursts from the pre-Swift era, which finds a high probability of association with old galaxies and old clusters (Gal-Yam et al. 2006). However, some degree of hesitation is introduced by the blue color and spiral nature of the host of GRB 050709; this may indicate some degree of heterogeneity among short-burst environments and progenitors (Villasenor et al. 2005; Hjorth et al. 2005; Fox et al. 2005). The importance of making a clear interpretation of class membership is that information about the burst progenitor has been mostly predicated on host galaxy characteristics. Clearer corroboration of class membership from the prompt emission would be helpful.

(We note that not all short bursts may be truly cosmological. The giant flare from SGR 1806-20 could have been detected by Swift to $\sim 50 \mathrm{Mpc}$, implying that eventually $\sim 5 \%$ of Swift yield for short bursts could be giant soft gamma repeater [SGR] events from the local neighborhood [Palmer et al. 2005].)

One intriguing complication to this developing picture is that GRBs 050709 and 050724 have softer, extended low-level emission, lasting for tens of seconds after the initial spikelike pulse. On the other hand, a long burst with one intense pulse at burst onset, separated by many seconds from nearly undetectable low-intensity pulses, might be mistaken for a short burst with extended emission. Figure 1 illustrates two such bursts. In this work we show that these cases can easily be distinguished from most of the "short" bursts, now believed to result from NSNS or NS-BH mergers. The initial double pulses ( $T_{90}$ durations $\sim 1.3$ and $1.8 \mathrm{~s}$ ) in both bursts shown in Figure 1 evidence clear spectral evolution (lower energies lagging higher energies), as do all long bursts for which counting statistics support a measurement of spectral lag (Norris 2002).

In contradistinction, a large majority of short bursts evidence negligible lag at BATSE energies (NSB01). This key discriminant, spectral lag, is examined in $\S 2$ for BATSE short bursts, in particular, the set of BATSE bursts with short initial spikes that are followed by extended, low-level emission; for the similar burst, GRB 050709 , recently detected by HETE-2; and for six short bursts detected by Swift's Burst Alert Telescope (BAT). We also examine the spectral hardness of the extended component and its dynamic range in intensity and fluence compared to

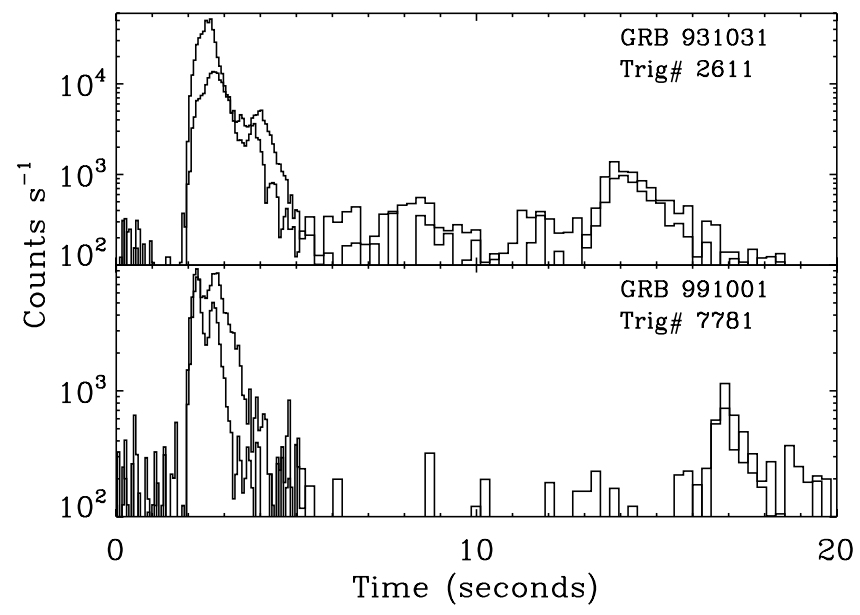

FIG. 1.-Time profiles on a logarithmic intensity scale for two BATSE long bursts for which the initial, intense emission has a duration $T_{90}<2 \mathrm{~s}$ and subsequent low-level emission extends the burst to $\sim 20 \mathrm{~s}$. The bin scale is $64 \mathrm{~ms}$ for the first $5 \mathrm{~s}$, then $1.024 \mathrm{~s}$. Thick line: $100-300 \mathrm{keV}$; thin line: $25-50 \mathrm{keV}$. The usual spectral evolution observed in long bursts is present in both the initial and extended parts of both bursts.

the initial spike. We discuss some ramifications of these findings in $\S 3$ and draw conclusions in $\S 4$.

\section{BURST CHARACTERISTICS}

We noticed early in the BATSE mission (e.g., GRBs 910709 and 921022), while fitting backgrounds to the four channel $64 \mathrm{~ms}$ DISCSC data, the presence of a small fraction of otherwise short bursts, but with extended lower level emission lasting for tens of seconds after the initial intense, short emission. We refer to this part of the burst as the "initial spike" - since this is the general appearance when binned on timescales longer than $\sim 50$ $100 \mathrm{~ms}$ - but in reality this interval (and generally in short bursts) sometimes comprises a group of a few closely spaced, very short episodes of emission (NSB01). At the end of the BATSE mission, we had accumulated about twenty candidates for a putative class that, defined solely in terms of duration, appeared to be an odd mix of short and long burst. The primary criterion for inclusion in this original set was that the initial spike was the dominant feature of the burst. About a quarter of these bursts were easily eliminated from further consideration on recognition that the later pulses were ordinary, but low-intensity, pulses that showed the usual organization in time and energy - evidencing longer decays at lower energies. Another quarter were too dim for profitable analysis. Eight bursts remained, and their temporal and spectral characteristics are examined here. The preparation and analysis procedures employed-including background fitting, duration, and spectral lag measurements - are described in previous works (Bonnell et al. 1997; NSB01; Norris 2002). Two remarks on these procedures are particularly pertinent to this work: We have assiduously reexamined our background fits several times for all BATSE bursts, allowing for the possibility of extended emission for all apparently short bursts. Also, where candidates for extended emission were identified, the BATSE signals were closely examined to ascertain that detector-to-detector count ratios were commensurate for initial spike and extended emission, and therefore qualitatively consistent with a common localization.

Time profiles of the remaining eight bursts are illustrated in Figure 2. The common interval is $100 \mathrm{~s}$, with the intensity rendered in the logarithm. The interval containing the initial spike is 


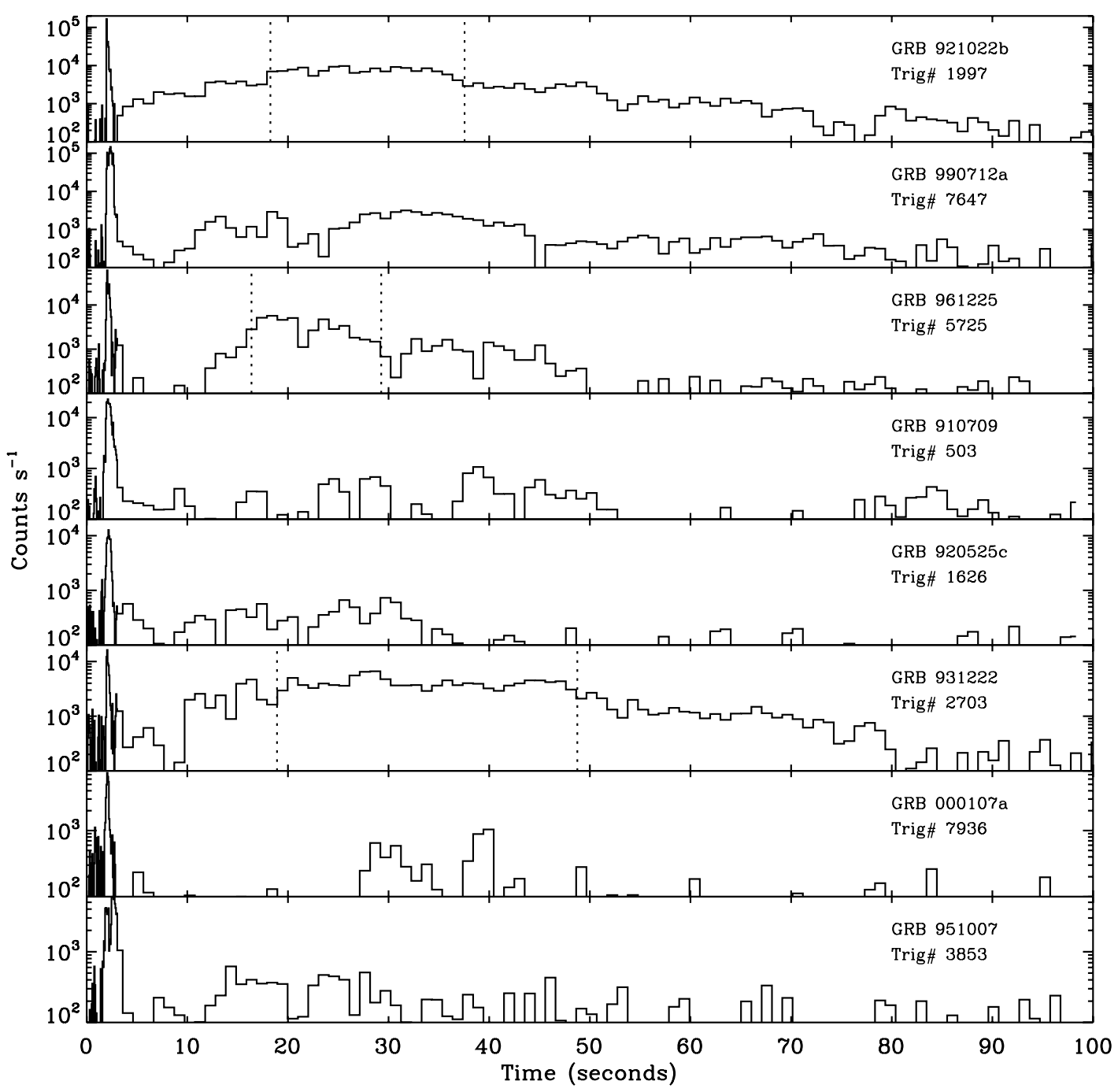

Fig. 2.-Same as Fig. 1, but for time profiles for the eight BATSE spikelike bursts analyzed in this work. Extended emission continues for up to $\sim 100 \mathrm{~s}$. The bursts are arranged top to bottom in order of decreasing intensity of the initial spike emission, which exhibits very little or negligible spectral evolution at BATSE energies. The tendency is evident for a hiatus to occur directly after the initial spike, followed by the rising extended emission, which is most prominent tens of seconds later. Vertical dotted lines in three profiles indicate intervals of extended emission analyzed for spectral evolution in $\S 2.2$.

binned at the native $64 \mathrm{~ms}$ resolution of the BATSE DISCSC data to allow a uniform comparison of the spike interval for all eight bursts, only four of which have higher time resolution TTE data. The remainder of each time profile is binned to $1.024 \mathrm{~s}$. From top down, the bursts are ordered by decreasing peak intensity of the initial spike as determined on the $64 \mathrm{~ms}$ timescale. The ordinate minimum in each panel is 100 counts $\mathrm{s}^{-1}$, representing $\sim 1 \sigma$ fluctuations above background level. Besides the solitary initial spike, the other salient feature is the extended, lowlevel emission, which tends to peak at levels $\sim 30-100$ times lower than the initial spike (except for GRB 931222, for which the peak intensity ratio is just a few). In binning up, some interesting spiky detail is lost for the bursts with more intense extended emission. In particular, any evidence for spectral evolution should be examined at the highest possible time resolution. This is addressed in $\S 2.2$ for the extended emission in the three most fluent and intense bursts (triggers 1997, 2703, and 5725). We now examine the spectral lag, hardness ratio, and duration for the spike emission in our sample of Figure 2 and compare to the distributions of these quantities for short BATSE bursts.

\subsection{Spectral Lag Analysis of Spikes}

In NSB01 a cross correlation function (CCF) approach was used to estimate spectral lags for BATSE short bursts. By fitting the CCF near its peak with a cubic, the native binning can be effectively overresolved by a factor of 2-4. The individual TTE photons were bootstrapped to obtain lag error estimates. We revisited the original analysis to ensure that no errant lag determinations were made. That is, infrequently a secondary, noncentral lobe of the CCF can be selected to be fitted in the automated process; secondary lobes arise when more than one significant peak is present. This undesired behavior was eliminated by constraining the process to fit the central lobe and then confirming visually that this occurred correctly for each of 101 bootstrap realizations for a given burst.

The initial spikes of these eight bursts in Figure 2 are related to the BATSE short-burst population in a fundamental way, as are the Swift and HETE-2 short bursts. The vast majority of short bursts have negligible spectral lags. Figure 3, adapted from NSB01, depicts peak flux versus lag, the latter measured between the BATSE energy channels $25-50$ and $100-300 \mathrm{keV}$ for 


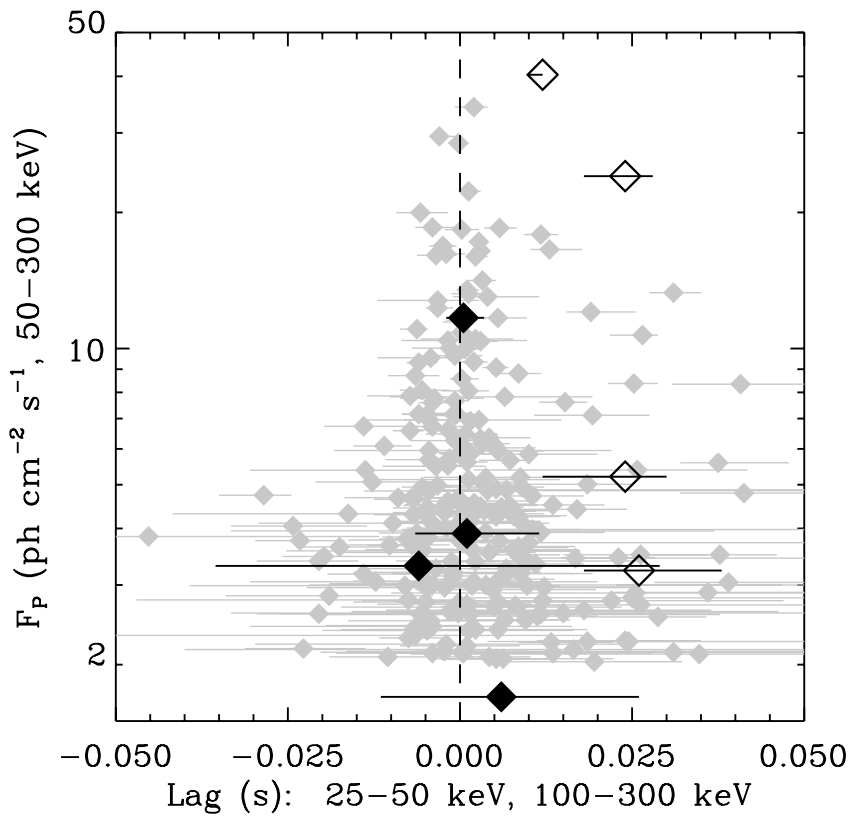

FIG. 3.-Peak flux (50-300 keV) vs. spectral lag (25-50 keV to $100-300 \mathrm{keV})$ for those 260 BATSE short bursts (gray diamonds) with time-tagged event (TTE) data that contained the burst. In addition, the lags for the spike emission for the bursts of Fig. 2 are plotted with filled black diamonds (TTE data binned at $8 \mathrm{~ms}$ ) or open diamonds (64 ms PREB+DISCSC data). Values for the latter set should be regarded as upper limits (see text). All (asymmetric) error bars are $\pm 1 \sigma$. Adapted from NSB01).

the 260 bursts for which the TTE data type contained essentially all the burst. The lags for BATSE short bursts, represented with gray diamonds and associated errors, appear to be distributed mostly symmetrically about zero lag.

In Figure 3 the lags for the initial spikes of the eight bursts of Figure 2 are shown as larger diamonds. The black filled diamonds are for the four bursts that were contained in the TTE data, which we binned to $8 \mathrm{~ms}$ to make the lag measurements. For the other four bursts, plotted with open diamonds, we measured the lags using PREB+DISCSC $64 \mathrm{~ms}$ data. While spectral lags in short bursts measured in the BATSE energy bands are usually consistent with zero, these bursts are nevertheless often asymmetric. The longer decays, at significantly lower intensity than the spike emission, develop more noticeably at lower energies. Because of its high intensity, the narrow spike would dominate the lag measurement if measured with sufficiently high time resolution, as in the four bursts measured with TTE data. More simply stated, when binned to $64 \mathrm{~ms}$ - longer than pulse durations in many short bursts - the weight of the spike intensity is lost, and the low-intensity decay at lower energies asserts a

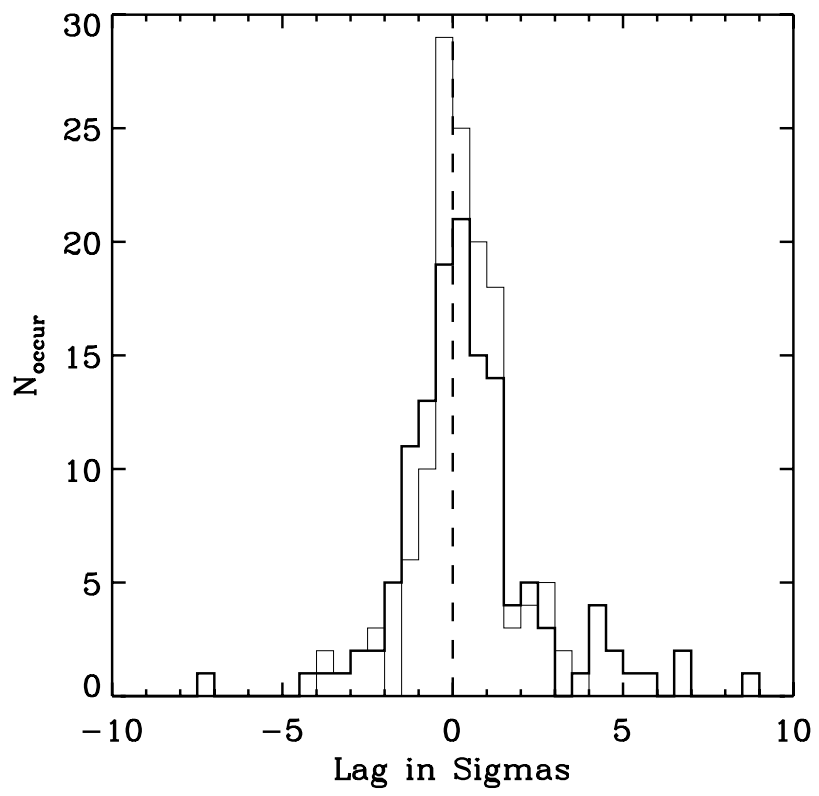

FIG. 4.- - Histograms of the lags for the original 260 short bursts of Fig. 3 rendered in sigmas. The thick (thin) histogram is that half of the sample with $F_{p}>(<) 4.25$ photons $\mathrm{cm}^{-2} \mathrm{~s}^{-1}$. Inspection of the time profiles reveals that the few negative outliers $(<-2.5 \sigma)$ appear to be valid, as do the larger number of positive outliers, which may represent the tail of the long-burst population. A large majority $(90 \%-95 \%)$ are consistent with zero lag.

measurable effect. This systematic lag-shifting effect, arising from finite binning on timescales longer than pulse durations, is readily demonstrated in simulations.

The GRB dates for the eight spikelike bursts with extended emission are listed in Table 1, along with the BATSE trigger number, peak flux $\left(F_{p}\right)$, duration, lag and associated errors, and the data type used to measure the lag ( $\mathrm{T}=\mathrm{TTE} 8 \mathrm{~ms}, \mathrm{D}=64 \mathrm{~ms})$. The lags should be compared with those of long bursts. Welldetermined, significantly positive lags for most bright long bursts span a dynamic range from $\sim 300$ down to $\sim 25 \mathrm{~ms}$ (see Fig. $2 b$ of Norris 2002), contiguous with the lags with the four largest values in Table 1, which also happen to be those measured with $64 \mathrm{~ms}$ data. Hence, these four bursts could represent the short tail of the long-burst distribution, but the systematic effect of the coarsely binned $64 \mathrm{~ms}$ data suggests that their lag measurements are effectively upper limits. Thus, as we argue below from additional convergent considerations, these four should probably also be categorized as short bursts.

To see if short bursts in the aggregate really have spectral lags consistent with zero, we plot in Figure 4 the distribution of lags in sigmas ( $1 \sigma=$ mean of the plus and minus errors) for the

TABLE 1

BATSE Bursts: Initial Spike Properties

\begin{tabular}{|c|c|c|c|c|c|c|c|}
\hline GRB Date & Trigger No. & $\begin{array}{c}F_{p} \\
\left(\mathrm{~cm}^{-2} \mathrm{~s}^{-1}\right)\end{array}$ & $\begin{array}{c}\text { Duration } \\
\text { (s) }\end{array}$ & $\begin{array}{c}\tau_{\text {lag }} \\
(\mathrm{ms})\end{array}$ & \multicolumn{2}{|c|}{$\begin{array}{l}\varepsilon_{\text {lag }} \\
(\mathrm{ms})\end{array}$} & Data Type \\
\hline 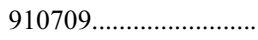 & 503 & 5.2 & 0.896 & 24.0 & +6.0 & -12.0 & $\mathrm{D}$ \\
\hline $920525 \ldots \ldots \ldots \ldots \ldots \ldots \ldots \ldots \ldots \ldots \ldots \ldots$ & 1626 & 3.2 & 0.528 & 26.0 & +12.0 & -8.0 & $\mathrm{D}$ \\
\hline $921022 \ldots \ldots \ldots \ldots \ldots \ldots \ldots \ldots \ldots \ldots \ldots \ldots \ldots$ & 1997 & 40.3 & 0.384 & 12.0 & +0.0 & -2.0 & $\mathrm{D}$ \\
\hline 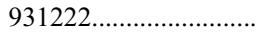 & 2703 & 3.9 & 1.776 & 1.0 & +10.5 & -7.5 & $\mathrm{~T}$ \\
\hline 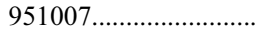 & 3853 & 3.3 & 1.392 & -6.0 & +35.0 & -29.5 & $\mathrm{~T}$ \\
\hline $961225 \ldots \ldots \ldots \ldots \ldots \ldots \ldots$ & 5725 & 11.7 & 1.216 & 0.5 & +3.0 & -2.5 & $\mathrm{~T}$ \\
\hline 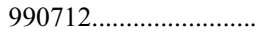 & 7647 & 24.1 & 0.648 & 24.0 & +4.0 & -6.0 & $\mathrm{D}$ \\
\hline 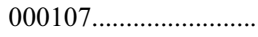 & 7936 & 1.7 & 0.368 & 6.0 & +20.0 & -17.5 & $\mathrm{~T}$ \\
\hline
\end{tabular}


260 short bursts analyzed in NSB01. The two histograms are plotted for the sample divided exactly in half at $F_{p}=4.25$ photons $\mathrm{cm}^{-2} \mathrm{~s}^{-1}$. The thick (thin) histogram represents the brighter (dimmer) half. Both subsets are closely distributed about zero lag. However, there is a handful $(\sim 7)$ of negative outliers $(<-2.5 \sigma)$, for which upon visual inspection we find that the low-energy channel (1) does appear to lead the high-energy channel (3). A larger number $(\sim 20$, where $1-2$ might be expected) have significant positive lags $(>+2.5 \sigma)$; these may be members of the tail of the long-burst distribution. We note that all outliers beyond $\pm 4 \sigma$ are in the brighter half of the sample (an effort to better characterize these significant outliers is in progress).

In summary, $90 \%-95 \%$ of the 260 apparently short bursts analyzed with TTE data have spectral lags consistent with zero, whereas, perhaps $\sim 5 \%-7 \%$ may actually be "intruders" from the long-burst distribution, having perhaps 1-2 close pulses (e.g., Fig. 1) that evolve spectrally at BATSE energies, as do all long bursts for which a significant measurement can be established (Norris 2002). In fact, the positive outliers mostly inhabit the top one fourth (0.3-2.0 s) of the duration distribution for this sample (NSB01). Pulses in short bursts tend to be an order of magnitude narrower than pulses in long bursts (e.g., Norris et al. 1994), and this would account for their lags being short, but not negligible. Since short bursts have a pulse width mode of $\sim 60 \mathrm{~ms}$ (FWHM), the brighter bursts in Figure 3 would evidence significantly positive lags if their $\tau_{\text {lag }} / \tau_{\text {width }}$ ratios were comparable to those in long bursts.

Thus, negligible spectral lag is the fourth attribute that makes the spike emission in short bursts really different from long bursts, in addition to their narrower pulses, the quasi-separation near $2 \mathrm{~s}$ in the duration distribution, and their higher hardness ratio in the aggregate. We use the same standard for hardness ratio as Kouveliotou et al. (1993), the ratio of total counts in the energy channels 3 and 2 (50-100 to $100-300 \mathrm{keV}), \mathrm{HR} 3 / 2$. Figure 5 illustrates HR $3 / 2$ versus the $T_{90}$ duration for our sample

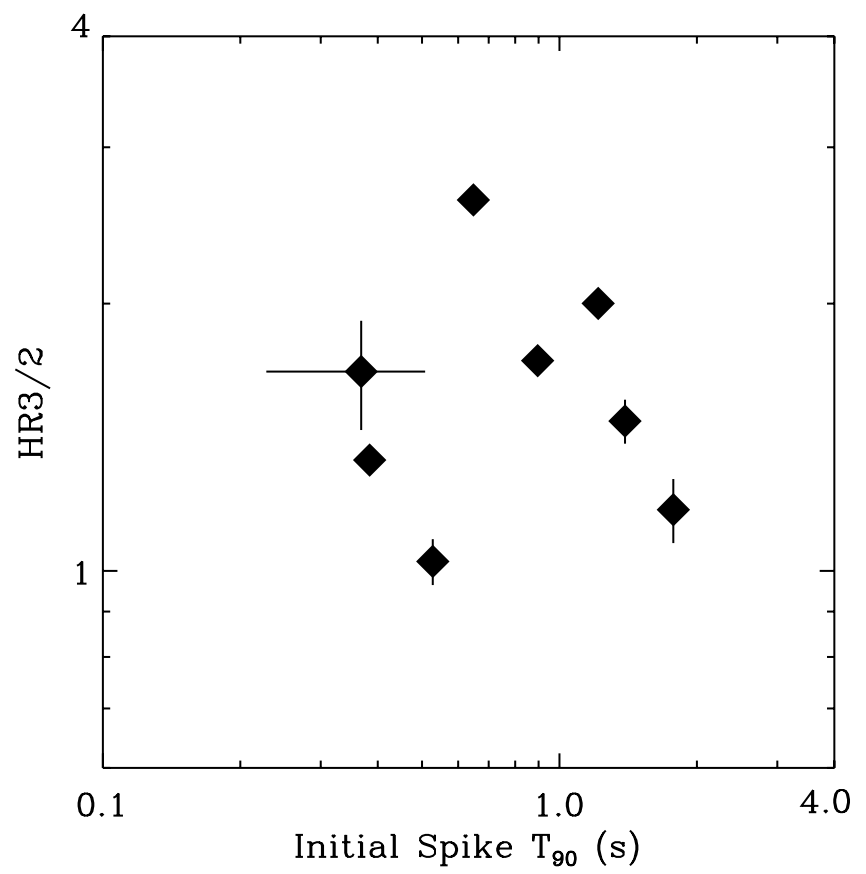

FIG. 5.-Counts spectral hardness ratio, HR3/2 [(100-300 keV)/(25-50 keV)], of the spike emission for the eight bursts of Fig. 2, vs. $T_{90}$ duration. Error bars $( \pm 1 \sigma)$ are shown but in some cases are smaller than the plot symbols. The sample falls within the dynamic ranges for short bursts as reported in Kouveliotou et al. (1993).

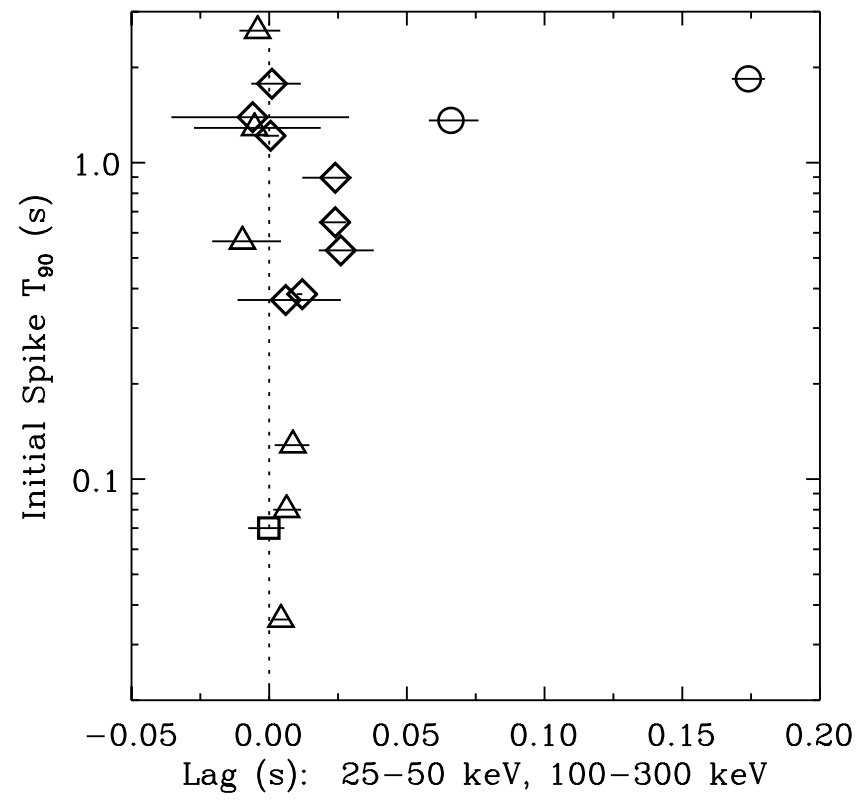

FIG. 6.-The $T_{90}$ duration vs. spectral lag for the spike emission in the eight BATSE bursts of Fig. 2 (diamonds) and the initial pulses of the two BATSE bursts in Fig. 1 (circles). Both sets shown with $\pm 1 \sigma$ errors. The results for six Swift BAT bursts are plotted (triangles) with $\pm 1 \sigma$ errors. Also plotted is a measurement of the lag for the spike emission of the HETE-2 burst (square, error bars: $3 \sigma$; see text), GRB 050709 .

of eight spike bursts with extended emission. The range in HR $3 / 2$ is $\sim 1-2.6$ with an average of 1.63 . This is comparable to the average of $\sim 1.5$ reported in Kouveliotou et al. over a full range of $\sim 0.25-3.0$. (Their HR3/2 average for long bursts is $\sim 0.9$.) The short-burst part of the duration distribution exhibits a broad maximum, $\sim 0.2-1.0 \mathrm{~s}$ (BATSE 3B catalog; Meegan et al. 1996). The median for the eight durations in Figure 5 is $\sim 0.75 \mathrm{~s}$, somewhat higher than for BATSE short bursts generally. However, the Konus sample of 130 short bursts peaks broadly between $\sim 0.1-$ $1.0 \mathrm{~s}$ (Mazets et al. 2002). Hence, our sample is within the expected dynamic ranges of HR3/2 and $T_{90}$ for short bursts.

It might be expected that as the spike duration increases, the lag would increase as well. Figure 6 illustrates duration versus lag for the eight BATSE short bursts with extended emission (diamonds). There is no trend apparent between these two parameters. Again, the four bursts with apparently positive lags were measured with $64 \mathrm{~ms}$ data, and their measurements should be considered as upper limits (see discussion above). For comparison, the lags and initial spike durations for the two long bursts of Figure 1 are also plotted (circles). As is obvious from Figure 1, their pulses are resolved at $64 \mathrm{~ms}$ resolution, and the lags are significantly positive. These two bursts represent the few, very shortest long bursts (formally, $T_{90}>2 \mathrm{~s}$ ) with low-level emission after an intense initial spike. However, their "extended" emission appears to have a different character than that of the eight bursts of Figure 2: the later episodes include one well-defined pulse per burst exhibiting the usual spectral evolution.

Also included in Figure 6 are the six short bursts detected by the Swift BAT for which event data are available and the burst was bright enough to make a spectral lag measurement. Table 2 lists the lag and duration measurements for these bursts. In four cases the lag was computed between BAT energy channels 3 and 1 (50100 and $15-25 \mathrm{keV}$ ), and in two cases between channels 4 and 2 (100-350 and 25-50 keV). Each choice was made to maximize the S/N level. The channel 4-2 pair corresponds nominally to the BATSE energy channels that were used for the measurements of 
TABLE 2

Swift BAT Bursts: Initial Spike Properties

\begin{tabular}{|c|c|c|c|c|c|c|}
\hline \multirow{2}{*}{$\begin{array}{c}\text { GRB Date } \\
050509 \mathrm{~b} \ldots \ldots \ldots \ldots \ldots \ldots \ldots \ldots \ldots \ldots \ldots \ldots \ldots\end{array}$} & \multirow{2}{*}{$\begin{array}{c}\text { Duration } \\
(\mathrm{s})\end{array}$} & \multirow{2}{*}{$\begin{array}{r}\begin{array}{r}\tau_{\text {lag }} \\
(\mathrm{ms})\end{array} \\
4.3\end{array}$} & \multicolumn{2}{|c|}{$\begin{array}{c}\varepsilon_{\text {lag }} \\
(\mathrm{ms})\end{array}$} & \multirow{2}{*}{$\frac{\text { Channel Pair }}{(31)}$} & \multirow{2}{*}{$\frac{\text { References }}{\text { Gehrels et al. (2005) }}$} \\
\hline & & & +3.2 & -3.0 & & \\
\hline 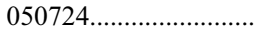 & $2.612 \pm 0.088$ & -4.2 & +8.2 & -6.6 & (31) & Barthelmy et al. (2005b) \\
\hline $050813 \ldots .$. & $0.564 \pm 0.054$ & -9.7 & +14.0 & -11.0 & (42) & Sato et al. (2005) \\
\hline $050925 \ldots \ldots$ & $0.128 \pm 0.006$ & 8.6 & +6.0 & -6.6 & (31) & Markwardt et al. (2005) \\
\hline 051105a ....................... & $0.080 \pm 0.012$ & 6.3 & +5.3 & -4.8 & (31) & Cummings et al. (2005) \\
\hline $051210 \ldots \ldots \ldots \ldots \ldots$ & $1.368 \pm 0.040$ & -5.3 & +24.0 & -22.0 & (42) & Barthelmy et al. (2005a) \\
\hline
\end{tabular}

Table 1. Our studies indicate that comparable lag measurements obtain for channel pairs shifted (quasi-) logarithmically, as is approximately the case here. For the four shortest bursts $\left(T_{90} \leq 0.25 \mathrm{~s}\right)$ the lags were measured with the event data binned to $4 \mathrm{~ms}$ resolution; for the two longer, dimmer bursts ( $T_{90}$ durations 0.6 and $1.2 \mathrm{~s}$ ) the data were binned to $32 \mathrm{~ms}$. In Figure 6 the results for BAT bursts are plotted with triangles. All are consistent with zero lag, irrespective of duration.

(The position of one of the six Swift bursts [GRB 050925] lies $0{ }^{\circ} .1$ from the Galactic plane, strongly suggesting that it is an SGR [Holland et al. 2005]. Ordinary SGR bursts also do not exhibit spectral evolution, although probably for a different reason than the cosmologically distant short-burst class [Golenetskii et al. 1984; Kouveliotou et al. 1987; Norris et al. 1991].)

The $T_{90}$ durations listed in Table 2 were measured using all the BAT event data in the energy band $15-350 \mathrm{keV}$, rather than burst signal counts generated by the mask-tagged background subtraction procedure. Our approach has the benefit of higher $\mathrm{S} / \mathrm{N}$, but the background level is less accurately determined. The penalty is small given that there is usually generous latitude for choosing the preburst background interval, and in each case we chose the postburst interval to end before the spacecraft slew commenced. The references in Table 2 give other estimates for the burst durations.

GRB 050709, detected by HETE-2, is similar to our sample of eight BATSE bursts - a short spike with extended emission (Villasenor et al. 2005). We digitized the time profiles of the spike emission in two of HETE-2's energy bands, 30-85 and 85$400 \mathrm{keV}$, from their Figure 3, and performed the same CCF lag analysis at the $4 \mathrm{~ms}$ resolution of these profiles. They report a duration for the spike of $70 \pm 10 \mathrm{~ms}(30-400 \mathrm{keV})$. The peak flux of the spike in the $50-300 \mathrm{keV}$ (same band as for BATSE bursts) was $\sim 20$ photons $\mathrm{cm}^{-2} \mathrm{~s}^{-1}$, bright enough to make a good lag measurement (cf. Table 1). The HETE-2 point (lag = $0.0_{-2.5}^{+2.0} \mathrm{~ms}, 1 \sigma$ formal error) is included in Figure 6 (square), but with liberal error bars $( \pm 3 \sigma)$, since we are unfamiliar with the particulars of their instrument and since the HETE-2 energy bands are contiguous and different than BATSE channels 1 and 3 (25-50 and 100-300 keV), which we use for the CCF analysis. Thus, the short bursts from the BATSE and BAT samples and the HETE-2 burst - with or without extended emission - all fit the paradigm for short bursts in general, in that their spectral lags are consistent with zero.

The apparently anomalous part of the picture is the extended emission present in one Swift burst and in the HETE-2 burst but discernible only in a handful of the $\sim 550$ short bursts detected by BATSE. We now examine the extended emission for the eight bursts in Figure 2.

\subsection{Extended Emission in Short Bursts}

All eight of the BATSE bursts in our sample have $T_{90}$ durations in the range 30-90 s, greater than the median for long bursts. The high counts ratio between the two componentsextended to the initial spike-makes for the long durations in our BATSE bursts. Because the extended emission component was readily apparent, they were previously assigned membership in the long-burst class. However, since the initial pulses have $T_{90}<$ $2 \mathrm{~s}$ (Fig. 6) in each case followed by a hiatus of several seconds before the extended component commences, the overall appearance is unlike that of most other long bursts. We consider the defining characteristic of the short-burst class to be negligible spectral evolution above $\sim 20 \mathrm{keV}$. This property of the initial spikes in our sample leads us to classify them as short bursts. Below we show that the same property obtains for the extended emission in the brightest cases.

Our BATSE sample represents the short bursts with the brightest extended emission, hence those with the highest $\mathrm{S} / \mathrm{N}$ level available for study. BATSE's unique combination of longevity (1991-2000) and large area allowed for the detection and examination of these few bursts. We emphasize that the norm for short bursts is very low-level (usually indiscernible) extended emission in an individual burst. Just as the bright end of the $\log \left[N\left(F_{p}\right)\right]-\log \left(F_{p}\right)$ distribution for GRBs becomes well populated only with sufficient exposure, our sample with intense extended components is available due to the 9 years of the Compton Gamma-Ray Observatory mission. In fact, all short bursts might have extended emission, but it is usually indistinguishable from background in individual bursts. Lazzati et al. (2001), Connaughton (2002), and Frederiks et al. (2004) did detect this component in the aggregate time profiles and reported that this persisted for tens of seconds and was softer than the initial spike.

The Swift and HETE-2 short bursts with extended low-level emission are similar in that the extended emission is softer than the initial spike (Barthelmy et al. 2005b; Villasenor et al. 2005). Mazets et al. (2002) also report a soft extended component (see their Table 3). The same is true for all eight bursts in our sample. Table 3 lists the ratio of total counts $>25 \mathrm{keV}$ of the two components (spike:extended), the HR3/2 measurements for spike and extended emission, and the ratio of the two HR3/2's. The extended emission is always spectrally softer than the spike.

The dynamic range of intensity of the two components is an important quantity for theories to entertain. In our eight BATSE bursts, the extended emission is relatively strong, with peak intensities only a factor of $2-10$ lower than the spike emission in three cases (compare Figs. 2 and 7). The two brightest extended components attain count rates of $\sim 10^{4} \mathrm{~s}^{-1}$. Assuming a typical background rate of $8000 \mathrm{~s}^{-1}$, a $1 \sigma$ fluctuation in Figure 2 is $\sim 100 \mathrm{~s}^{-1}$. Lazzati et al. (2001) detect the extended component in a sample of 76 co-added bursts with the averaged time profile binned to $16 \mathrm{~s}$ samples. In their Figure 1 a $1 \sigma$ fluctuation translates to $\sim\left(8000 \mathrm{~s}^{-1} \times 16 \mathrm{~s} \times 76\right)^{1 / 2} \approx 3200$, or $2.5 \mathrm{~s}^{-1}$ burst $^{-1}$ in the average. Thus, the dynamic range in intensity for the extended component among BATSE bursts is $>4000$, since some of 
TABLE 3

Extended Emission Compared to Initial Spike

\begin{tabular}{|c|c|c|c|c|c|c|c|}
\hline \multirow[b]{2}{*}{ GRB Date } & \multirow{2}{*}{$\begin{array}{l}\text { Counts Ratio } \\
\qquad(\mathrm{S} / \mathrm{E})^{\mathrm{a}}\end{array}$} & \multicolumn{2}{|c|}{ SPIKE } & \multicolumn{2}{|c|}{ EXTENDED } & \multicolumn{2}{|c|}{ EXTENDED:SPIKE } \\
\hline & & $\mathrm{HR} 3 / 2$ & $\varepsilon_{\mathrm{HR}}$ & $\mathrm{HR} 3 / 2$ & $\varepsilon_{\mathrm{HR}}$ & $R-\mathrm{HR}^{\mathrm{b}}$ & $\varepsilon_{\mathrm{R}-\mathrm{HR}}$ \\
\hline 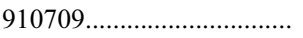 & 0.815 & 1.725 & 0.052 & 0.840 & 0.102 & 0.487 & 0.061 \\
\hline 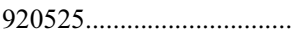 & 0.500 & 1.025 & 0.061 & 0.748 & 0.097 & 0.730 & 0.104 \\
\hline 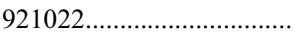 & 0.077 & 1.333 & 0.036 & 0.773 & 0.012 & 0.580 & 0.018 \\
\hline 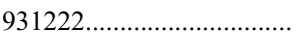 & 0.023 & 1.172 & 0.097 & 1.070 & 0.019 & 0.913 & 0.077 \\
\hline 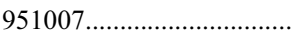 & 0.461 & 1.475 & 0.084 & 0.421 & 0.079 & 0.285 & 0.056 \\
\hline 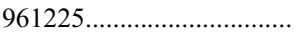 & 0.197 & 2.001 & 0.072 & 1.140 & 0.043 & 0.570 & 0.030 \\
\hline 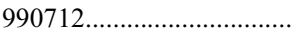 & 0.902 & 2.617 & 0.033 & 0.906 & 0.041 & 0.346 & 0.016 \\
\hline 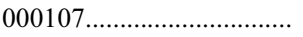 & 0.397 & 1.677 & 0.236 & 1.298 & 0.277 & 0.774 & 0.198 \\
\hline
\end{tabular}

${ }^{\text {a }}$ Ratio of emission in the spike to the extended emission.

b Ratio of the extended emission hardness ratio to the spike emission hardness ratio.

the bursts in the Lazzati et al. sample must be dimmer than the average. At least 30 BATSE short bursts with $F_{p}>10$ photons $\mathrm{cm}^{-2} \mathrm{~s}^{-1}$ show no individual evidence of extended emission, whereas three of our sample have comparable peak fluxes (see Fig. 3). The initial spike in GRB 931222 is $\sim 2$ times more intense than the peak in the extended emission. We conclude that the dynamic range in the ratio of peak intensities, spike:extended, is $\sim 10^{4}$.

The range in the ratio of total counts is also large. For our BATSE sample, the dynamic range of the ratio, spike:extended,

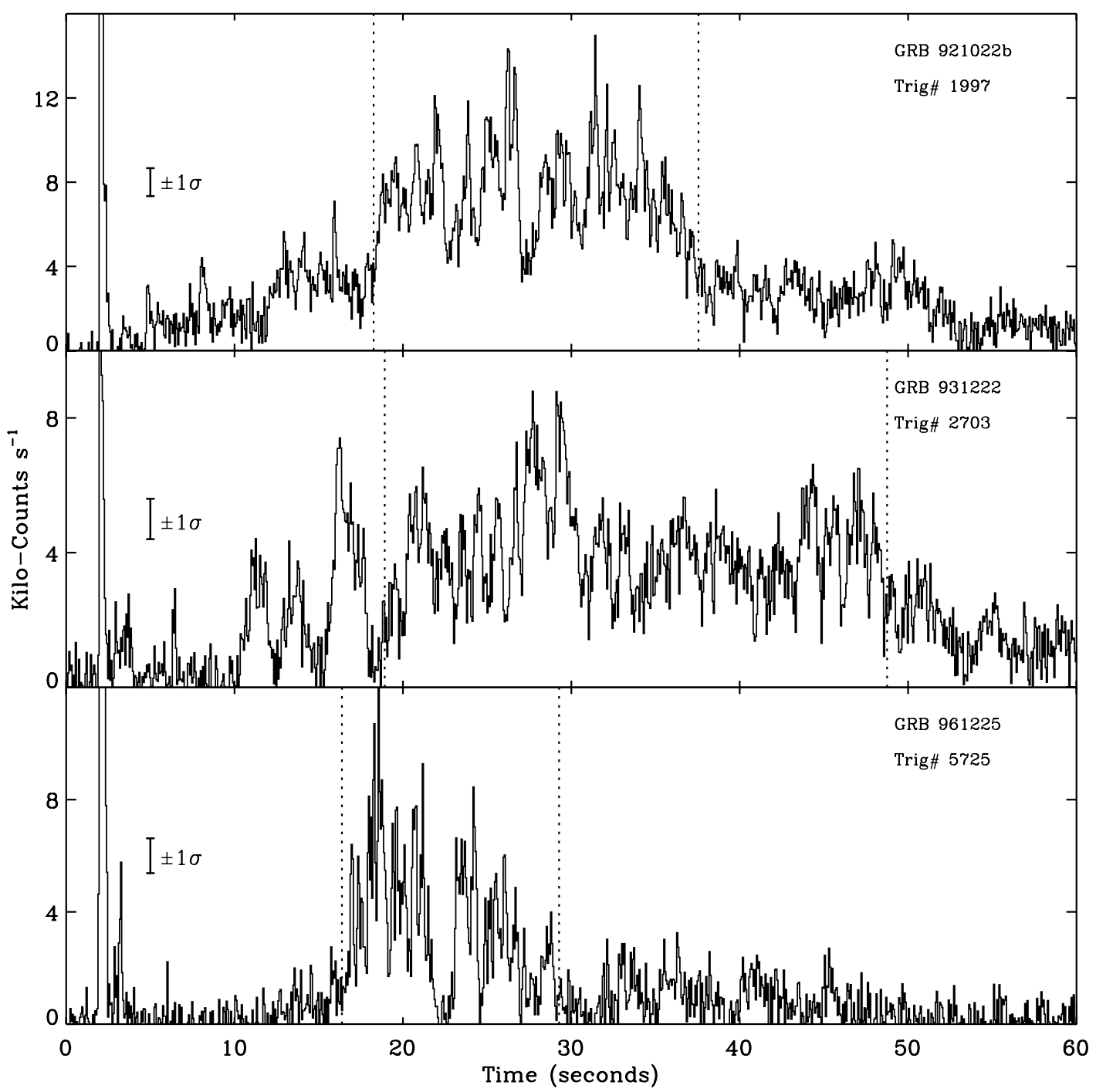

FIG. 7.-Time profiles for the three bursts of Fig. 2 with the brightest and most fluent extended emission. The vertical axis truncates the initial spike to emphasize

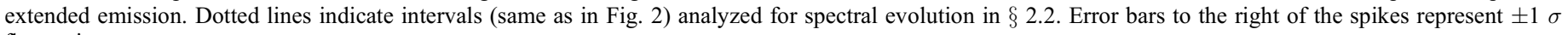
fluctuations. 
is $\sim 50: 1$ (see Table 3). The HETE-2 burst, GRB 050709, also exhibited more than an order of magnitude more counts in the extended emission at low energies, $2-25 \mathrm{keV}$ (Villasenor et al. 2005). However, the initial spike sometimes dominates. The Konus experiment on the Wind spacecraft detected 11 (out of 130 total) short bursts that individually exhibited extended emission (Mazets et al. 2002; Frederiks et al. 2004). Mazets et al. report fluences for the two components. The fluence ratios tend to be a factor of several lower than our count ratios in Table 3, even after taking into account the difference in hardness ratios of the two components. Since in most bursts the extended emission is detected only in the aggregate, the same reasoning as described above for the range in flux ratio applies for the overall dynamic range of the total counts (or fluence) ratio, which must also be of order $10^{4}$. Thus, physical models must account for how the division in the energy budget between the two components can vary so greatly from burst to burst.

The extended component is visible in only $\sim 2 \%$ of BATSE short bursts, but it appears that Konus, Swift, and HETE-2 may be detecting a significantly larger fraction of this softer emission. At least two factors may contribute to the higher yields of these two missions. The BATSE Large Area Detectors were sensitive down to $\sim 25 \mathrm{keV}$ and viewed the background over a large portion of the sky. The response for Konus continues to $\sim 12 \mathrm{keV}$, Swift's BAT to $\sim 15 \mathrm{keV}$ (Barthelmy et al. 2005c), and HETE-2 to $2 \mathrm{keV}$ (Ricker et al. 2003). For Swift, the mask-tagged background subtraction method applied to event data yields a time profile with effectively zero signal (but nonzero variance) integrated over directions different than the burst direction, allowing sensitive searches for extended hard X-ray emission from bursts.

An inspection of Figure 2 reveals that the extended emission usually does not commence directly succeeding the spike. Rather, after a hiatus of $\sim 10 \mathrm{~s}$ the low-level emission builds later to a relatively broad maximum on a timescale of $30-50 \mathrm{~s}$. When the count statistics support examination at $64 \mathrm{~ms}$ resolution, the emission is very spiky, as shown in Figure 7 for the three bursts (triggers 1997, 2703, and 5725) with the most intense extended emission. We carried out the CCF analysis for these bursts using the intervals bounded by pairs of dashed lines in Figure 2 (the other three bursts were too weak to give constraining results). Outside the selected intervals, the emission drops off to successively lower valleys between spiky episodes; inclusion of these lower intensity regions tends to increase the error estimates. The results are summarized in Table 4. The spectral lags for extended emission in two bursts are $16_{-8}^{+12}$ and $12_{-6}^{+16} \mathrm{~ms}$, formally $2 \sigma$ different from zero lag, and consistent with zero for one burst. The central values for the positive lags are comparable to or less than the lags measured for spikes in four bursts of our sample (compare with Fig. 3 and Table 3). Determining whether or not the extended emission in such bursts would usually be consistent with zero when resolved at finer time resolution - as we found to be often the case for the spike emission-will require, e.g., bright Swift bursts examined using time- and energy-

TABLE 4

Extended Emission Spectral Lag

\begin{tabular}{crrrr}
\hline \hline GRB Date & $\begin{array}{c}\tau_{\text {lag }} \\
(\mathrm{ms})\end{array}$ & $\begin{array}{c}\varepsilon_{\mathrm{lag}} \\
(\mathrm{ms})\end{array}$ & $\begin{array}{c}\varepsilon_{\mathrm{lag}} \\
(\mathrm{ms})\end{array}$ & \multicolumn{1}{c}{$\begin{array}{c}\text { Interval } \\
(\mathrm{s})\end{array}$} \\
\hline $921022 \ldots \ldots \ldots \ldots \ldots .$. & 16.0 & +12.0 & -8.0 & $18.3-37.6$ \\
$931222 \ldots \ldots \ldots \ldots \ldots .$. & 8.0 & +20.0 & -22.0 & $18.9-48.8$ \\
$961225 \ldots \ldots \ldots \ldots \ldots \ldots . .$. & 12.0 & +16.0 & -6.0 & $16.4-29.3$ \\
\hline
\end{tabular}

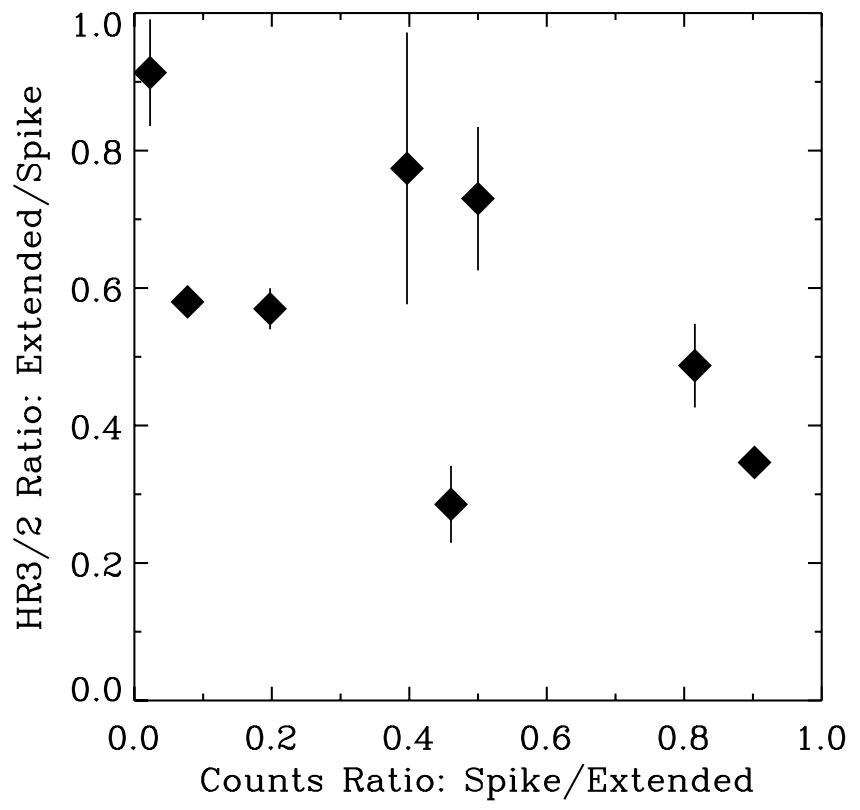

FIG. 8. - Ratio of HR3/2 (spike:extended) vs. total counts ( $>25 \mathrm{keV}$ ) ratio, extended:spike. There is a slight hint that when the extended emission is relatively less fluent, it tends to be spectrally softer.

tagged event data. In the case of GRB 050709, Villasenor et al. (2005) report no spectral evolution for the extended emission.

Finally, we ask if there is a relationship between strength of the extended emission and its spectral hardness, relative to the spike emission. Figure 8 illustrates the total counts ratio versus the ratios of HR3/2. The hint of a possible trend is apparent: when the extended emission is relatively less fluent, it may tend to be spectrally softer. A larger sample would be necessary to support this loose correlation.

\section{DISCUSSION}

In summary, our spectral lag analysis for eight BATSE short bursts with extended emission shows that a fundamental defining characteristic of the short-burst class is that the initial spike exhibits negligible spectral lag at energies above $\sim 25 \mathrm{keV}$. This behavior is nearly ubiquitous for the 260 bursts with $T_{90}<2 \mathrm{~s}$ for which the BATSE TTE data type completely included the initial spike: Their spectral lags measured between the $25-50$ and $100-$ $300 \mathrm{keV}$ energy ranges are consistent with zero in $90 \%-95 \%$ of the cases. Most outliers have positive lags and probably represent the tail of the long-burst distribution. Similarly, our analyses of six Swift BAT short bursts and one HETE-2 burst (Villasenor et al. 2005) show that their initial spikes exhibit negligible spectral lag. Extended emission was evident in the HETE-2 burst (GRB 050709; Villasenor et al. 2005) and one of the Swift bursts (GRB 050724; Barthelmy et al. 2005b). Thus, membership for short bursts with extended emission - which can sometimes extend their formal $T_{90}$ measurements to $>2 \mathrm{~s}-$ appears decided within the short-burst class.

The extended emission in our sample of eight BATSE short bursts is always softer than the spike emission, as was reported for all such bursts observed by HETE-2, Swift, and Konus (Villasenor et al. 2005; Barthelmy et al. 2005b; Mazets et al. 2002) and for averaged time profiles of BATSE short bursts (Lazzati et al. 2001; Connaughton 2002). Considering all these studies combined with our results, we find that the dynamic ranges in both flux and fluence ratios, spike:extended, is very 
large, of order $10^{4}$. How the apportioning of the energy budget may vary so greatly between the two components is a major question. We also find that when the extended emission is sufficiently intense to make a measurement, its appearance is very spiky and the average spectral lag is negligible, similar to that of the initial spike.

The resulting canonical picture of "short hard" bursts is modified in several ways. First, when accompanied by sufficiently fluent extended emission, short bursts are not always shorter than $\sim 2 \mathrm{~s}$; they can have $T_{90}$ durations lasting tens of seconds. Second, as detailed in $\S 1$, the appearance that short bursts are spectrally harder than long bursts may be partially or completely attributable to spectral and temporal trigger selection effects. Third, when sufficiently bright to support examination on short timescales, the extended emission has a spiky pulselike appearance and thus may not be a standard "afterglow." Instead, these pulses might be analogous to the X-ray flares observed accompanying the prompt portions of afterglows as revealed by Swift's $\mathrm{X}$-Ray Telescope and attributed to central engine activity (Zhang et al. 2006). Fourth, in many cases in which the extended component is discernible in an individual burst (Figs. 2 and 7; Villasenor et al. 2005; Barthelmy et al. 2005a, 2005b, 2005c) there is a hiatusan interval of negligible or low-intensity emission - after the initial spike and before the extended emission strengthens appreciably. This frequently occurring feature has no obvious analog in long bursts. However, the hiatus is not always present, as can be appreciated from the Konus Catalog of Short Bursts (Mazets et al. 2002). The absence of a hiatus in individual bursts and in the average profile of BATSE short bursts (Lazzati et al. 2001) may be evidence against models involving ejecta from the source impinging on a companion star (see below).

It remains to explain the negligible spectral lags that are characteristic of short bursts at energies above $\sim 15 \mathrm{keV}$, in contrast to the signature of positive lags in long bursts (Norris 2002). Recall that pulses in short bursts are $\sim 10-20$ times shorter than in most long bursts (NSB01). A lower limit on pulse duration due to relativistic beaming is $\tau_{\text {rel }} \sim(1+z) R / 2 c \Gamma^{2}$ (angular spreading, or the "curvature effect"), where $R$ is the distance of the emission region from the source and $\Gamma$ is the bulk Lorentz factor of the emitting matter (Fenimore et al. 1996; Sari \& Piran 1997). Later emission that arrives during the interval $\tau_{\text {rel }}$ from further off axis is lower frequency - this is one inescapable component of spectral lag. In short bursts $\Gamma$ must be sufficiently large so that $\tau_{\text {rel }}$ is negligible compared to our instruments' measurement capabilities. In fact, $\tau_{\text {rel }}$ must be significantly shorter than the pulse timescale, or else we would be able to measure its contribution to spectral lag in the brighter short bursts (as discussed in $\S 2.1$ ). Therefore, the (dominant) timescale of pulses in short bursts must be attributable to variability of central engine activity, rather than to relativistic beaming. Then to avoid a significant contribution to spectral lag from $\tau_{\text {rel }}$, the Lorentz factors in short bursts must be several times higher than in long bursts.

Assuming that $\tau_{\text {rel }}$ is a major contribution to lag in both long and short bursts, we can estimate roughly the average $\Gamma$ in short bursts relative to that in long bursts, as follows. Above a peak flux threshold of $F_{p}>10$ photons $\mathrm{cm}^{-2} \mathrm{~s}^{-1}$, the lag measurements for both samples are usually accurately measured. The median lag for the 90 BATSE long bursts above this threshold is $\sim 48 \mathrm{~ms}$ (Norris 2002), and for the same threshold the average lag for 30 BATSE short bursts (excluding the few outliers deemed to be long-burst intruders) is $0.1 \pm 3.0 \mathrm{~ms}(1 \sigma)$. Since their lag distribution is near Gaussian, we take the $2 \sigma$ sample error, $2 \times 3.0 \mathrm{~ms} /(30-1)^{1 / 2} \approx 1 \mathrm{~ms}$, to be the effective upper limit for the short-burst sample lag. If the other factors con- tributing to $\tau_{\text {rel }}(R$ and $1+z)$ were comparable for long and short bursts, then we would have

$$
\Gamma_{\text {short }} / \Gamma_{\text {long }} \sim\left(\tau_{\text {rel_long }} / \tau_{\text {rel_short }}\right)^{1 / 2}>6.8 .
$$

To take into account the different (observed) redshift distributions of the two classes, the rough estimate in equation (1) should be reduced by $\delta \sim\left[\left(1+z_{\text {long }}\right) /\left(1+z_{\text {short }}\right)\right]^{1 / 2}$. Suppose the median short- and long-burst redshifts are $z_{\text {short }} \sim 0.5$ and $z_{\text {long }} \sim 2.5$. Then with $\delta \sim 1.5, \Gamma_{\text {short }} / \Gamma_{\text {long }}>4.5$. Some estimates for $\Gamma_{\text {long }}$ are of order 100-200 (e.g., Zhang et al. 2006). Then, modulo the factor due to the emission distances from the source, $\left(R_{\text {long }} / R_{\text {short }}\right)^{1 / 2}$, we would have $\Gamma_{\text {short }} \sim 500-1000$. Similar Lorentz factors are predicted from modeling with general relativistic hydrodynamic codes for outflows from compact object mergers (Aloy et al. 2005). Note that for $R=3 \times 10^{13} \mathrm{~cm}$, $\Gamma=10^{3}$, and $z=1$, angular spreading gives $\tau_{\text {rel }}=1 \mathrm{~ms}$.

At energies sufficiently lower than the BATSE bandpass $(<25 \mathrm{keV})$, short-burst pulses may exhibit longer decays - and therefore significant lags - due to the curvature effect. A good example is GRB 050709, for which the initial spike emission clearly becomes asymmetric below $10 \mathrm{keV}$, as revealed by HETE-2 (see Fig. 3 in Villasenor et al. 2005).

Like the spike emission, the bright, fluent extended emission in three bursts exhibits negligible spectral lag. The general inference is that both emission components may arise in the same region, but (possibly) from different mechanisms; if the extended component were generated at a significantly larger distance from the source than the spike component, then energy-dependent arrival-time dispersion would probably be manifest, due to the kinematic correlation between energy and off-axis angle in relativistic beaming.

MacFadyen et al. (2005) propose a model for XRFs in burst afterglows, relying on the interaction of the relativistic outflow from an accreting neutron star and a noncompact stellar companion at a separation of approximately a light-minute. Such a scenario clearly has some properties consistent with the extended emission examined here, peaking $\sim 30-50 \mathrm{~s}$ after the initial spike. For such a binary progenitor, a hiatus between spike and extended emission, as well as flux in the extended emission, would be expected to correlate with separation between the NS and stellar companion. Absence of a hiatus, as apparent for some bursts in the Konus Catalog of Short Bursts (Mazets et al. 2002), could rule out this model for short bursts in general. Constrained by the binary separation, the interaction region would be compact enough to exhibit negligible geometrically induced lags at gamma-ray energies. The observed spiky structure of the extended emission would suggest a patchy outflow with a filling factor $\sim 50 \%$ to produce constituent pulses of width $\sim 250-1000 \mathrm{~ms}$, as observed.

\section{CONCLUSIONS}

We have presented strong evidence-including results from BATSE, HETE-2, and Swift samples - that as a class, short GRBs have negligible spectral lag at energies above $\sim 15-25 \mathrm{keV}$. The average lag (25-50 to $100-300 \mathrm{keV})$ for the 30 brightest BATSE short bursts is $\sim 0.1 \pm 0.5 \mathrm{~ms}$, compared to $\sim 50 \mathrm{~ms}$ for bright long bursts. Lorentz factors several times higher than inferred for long bursts would obtain for short bursts $(\Gamma \sim 500-1000)$ if a major portion of spectral lag is attributable to relativistic beaming. However, the existence of lags when lower energy bands are considered is not precluded (for example, GRB 050709; Villasenor et al. 2005), since the angular dependence of the "curvature effect" produces longer delays at lower energies. 
We show that the extended component - always softer than the initial spike - can manifest a dynamic range in intensity and fluence of order $\sim 10^{4}$ compared to the initial spike. Infrequently, as in our BATSE sample of eight bursts, the strength of the extended emission converts an otherwise short burst into one with a duration that can be tens of seconds, making it appear to be a long burst. How the short-burst mechanism can give rise to such a range in total energy in the extended emission is unclear.

We also noted that spectral hardness is not an uniquely defining characteristic for short bursts. Their extended emission is softer, whereas long bursts - at onset as hard as a short burst's initial spikes - tend to soften as the burst progresses. Also, a specific instrument's trigger criteria impose strong selection effects, masking the actual distribution of spectra in a parent population. For instance, XRFs were essentially nonexistent in the BATSE sample but constitute one third of the HETE-2 sample (Sakamoto et al. 2005). Thus, "short hard" bursts are neither necessarily short, nor do they deserve to be labeled as strictly harder than long bursts. We suggest that the current popular nomenclature for the two classes, SHB (for short hard burst) and LSB (for long soft burst), is at best misleading.

However, short bursts do have pulses that are $\sim 10-20$ times shorter than pulses in long bursts (NSB01). The second clear distinction between the two classes that we have emphasized here is spectral lag. These two properties of the prompt emission in short bursts may enable us to discern which class a given burst belongs to, especially when host type, immediate environment, and afterglow information are indecisive.

We are grateful to the anonymous referee for suggestions and corrections that significantly contributed to the substance and organization of this work. We thank Neil Gehrels and Bing Zhang for helpful conversations and encouragement.
Aloy, M. A., Janka, H.-T., \& Muller, E. 2005, A\&A, 436, 273

Barthelmy, S. D., et al. 2005a, GCN Circular, 4321, 1, http://gcn.gsfc.nasa.gov/ $\mathrm{gcn} / \mathrm{gcn} 3 / 4321 . \mathrm{gcn} 3$

- 2005b, Nature, 438, 994

. 2005c, Space Sci. Review, 120, 143

Bloom, J., et al. 2006, ApJ, 638, 354

Bonnell, J. T., Norris, J. P., Nemiroff, R. J., \& Scargle, J. D. 1997, ApJ, 490, 79

Briggs, M. S., et al. 1996, ApJ, 459, 40

Connaughton, V. 2002, ApJ, 567, 1028

Cummings, J., et al. 2005, GCN Circular, 4190, 1, http://gcn.gsfc.nasa.gov/gcn/ $\operatorname{gcn} 3 / 4190 . \mathrm{gcn} 3$

Fenimore, E. E., in't Zand, J. J. M., Norris, J. P., Bonnell, J. T., \& Nemiroff, R. J. 1995, ApJ, 448, L101

Fenimore, E. E., Madras, C., \& Nayakshin, S. 1996, ApJ, 473, 998

Fox, D. B., et al. 2005, Nature, 437, 845

Frederiks, D. D., et al. 2004, in ASP Conf. Ser. 312, Gamma-Ray Bursts in the Afterglow Era, ed. M. Feroci et al. (ASP: San Francisco), p. 197

Gal-Yam, A., et al. 2006, ApJ, submitted (astro-ph/0509891)

Gehrels, N., et al. 2005, Nature, 437, 851

Ghirlanda, G., et al. 2004, A\&A, 422, L55

Golenetskii, S. V., Il'inskii, V. N., \& Mazets, E. P. 1984, Nature, 307, 41

Hjorth, J., et al. 2005, Nature, 437, 859

Holland, S. T., et al. 2005, GCN Circular, 4034, 1, http://gcn.gsfc.nasa.gov/gcn/ gen3/4034.gcn3

Kouveliotou, C., et al. 1987, ApJ, 322, L21

1993, ApJ, 413, L101

Lamb, D. Q., \& Reichart, D. E. 2000, ApJ, 536, 1

Lazzati, D., Ramirez-Ruiz, E., \& Ghisellini, G. 2001, A\&A, 379, L39

Lee, T. T., \& Petrosian, V. 1996, ApJ, 470, 479

\section{REFERENCES}

MacFadyen, A. I., Ramirez-Ruiz, E., \& Zhang, W. 2005, preprint (astro-ph/ 0510192)

Mallozzi, R. S., et al. 1995, ApJ, 454, 597

Markwardt, C., et al. 2005, GCN Circular, 4037, 1, http:/gcn.gsfc.nasa.gov/ $\mathrm{gcn} / \mathrm{gcn} 3 / 4037 . \mathrm{gcn} 3$

Mazets, E. P., et al. 2002, Konus Catalog of Short GRBs (St. Petersburg: Ioffe LEA), http://www.ioffe.ru/LEA/shortGRBs/Catalog/

Meegan, C. A., et al. 1996, in AIP Conf. Proc. 384, Gamma-Ray Bursts, ed. C. Kouveliotou, M. F. Briggs, \& G. J. Fishman (New York: AIP), 291

Norris, J. P. 2002, ApJ, 579, 386

Norris, J. P., Hertz, P., Wood, K. S., \& Kouveliotou, C. 1991, ApJ, 366, 240

Norris, J. P., Nemiroff, R. J., Davis, S. P., Kouveliotou, C., Fishman, G. J., Meegan, C. A., \& Paciesas, W. S. 1994, in AIP Conf. Proc. 307, Gamma-Ray Bursts, ed. G. J. Fishman, J. J. Brainerd, \& K. Hurley (New York: AIP), 172

Norris, J. P., Scargle, J. D., \& Bonnell, J. T. 2001, in Gamma-Ray Bursts in the Afterglow Era, ed. E. Costa, F. Frontera, \& J. Hjorth (Berlin: Springer), 40 (NSB01)

Norris, J. P., et al. 1996, ApJ, 459, 393

Palmer, D. M., et al. 2005, Nature, 434, 1107

Ricker, G. R., et al. 2003, in AIP Conf. Proc. 662, Gamma-Ray Burst and Afterglow Astronomy 2001, ed. G. R. Ricker \& R. Vanderspek (New York: AIP), 3

Sakamoto, T. 2006, in Gamma Ray Bursts in the Swift Era, ed. S. S. Holt, N. Gehrels, \& J. Nousek (New York: AIP), in press

Sakamoto, T., et al. 2005, ApJ, 629, 311

Sari, R., \& Piran, T. 1997, ApJ, 485, 270

Sato, G., et al. 2005, GCN Circular, 3793, 1, http:/gcn.gsfc.nasa.gov/gcn/gcn3/ 3793.gen3

Villasenor, J. S., et al. 2005, Nature, 437, 855

Zhang, B., et al. 2006, ApJ, 642, 354 